\title{
Pyridoxine-dependent seizures: an atypical case
}

\author{
Rajesh RamachandranNair ${ }^{1}$, Vellani T. Ajithkumar ${ }^{2}$ \\ Departments of ${ }^{1}$ Neurology and ${ }^{2}$ Pediatrics, Medical College Hospital, Calicut, India
}

Pyridoxine-dependent seizure (PDS) is a readily treatable cause of intractable seizures in childhood (1). Not more than 100 cases have been reported in the English language indexed journals. Delay in initiation of treatment could result in permanent mental handicap. It is generally recommended that a trial of pyridoxine should be given in all cases of intractable seizures below the age of 3 years (2). We report a case of 4-year-old boy with mental retardation and intractable seizures who, responded well to pyridoxine. This report emphasizes the importance of considering PDS in children with intractable seizures beyond 3 years.

A 4-year-old boy was admitted to the pediatrics intensive care unit with status epilepticus. He was born to non-consanguineous parents with an Apgar score of 10 at 5 minutes. There was no family history of seizures or mental impairment. Child attained social smile at 4 months, turned over at 7 months, walked independently at 20 months, and started saying 2-3 words at 3 years. Generalized seizures (partial seizures with generalization and myoclonus) started at the age 2 years. Various antiepileptic drugs either as monotherapy or in combination resulted mild reduction in seizure frequency. At 4 years he could speak 3-4 words, could indicate toilet needs and walk with minimal difficulty. His vision and hearing were normal. Electroencephalogram (EEG) showed multifocal spike and wave discharges. MRI of brain showed mild diffuse cerebral atrophy with prominent ventricles. The following tests were normal or negative: hemogram, serum calcium and phosphorous, blood thyroid hormone profile, renal and liver function tests, serum ammonia, blood arterial $\mathrm{pH}$, plasma organic acids, and urine homocysteine, reducing substances and organic acids. He was continued

Correspondence: Rajesh RamachandranNair M.D., DM, Pediatric Neurologist, Department of Neurology,

Medical College Hospital,

Calicut, 673008 India.

Tel: 91495 2359681, fax: 914952358409

E-mail: drrajeshram@rediffmail.com

Received: February 17, 2004.

Accepted: March 16, 2004. on valproate and phenytion. He developed status epilepticus without drug default or fever. The cerebrospinal fluid analysis was normal. Intravenous phenytoin, phenobarbitone and midazolam failed to control status. EEG showed continuous spike and wave discharges. He was started on $40 \mathrm{mg}$ of oral pyridoxine per day as a therapeutic trial. Within 48 hours he became seizure free. The repeated EEG showed complete disappearance of epileptiform activity. All the antiepileptic drugs were stopped in 2 weeks without recurrence of seizures. He reached the previous level of psychomotor functioning. Pyridoxine was stopped for 3 days after obtaining parents' consent. Generalized seizures (four attacks) occurred on the 4th day. Pyridoxine was restarted in the same dose and he became seizure free the next day. Child remained seizure free till the last follow up, which was eight months after the diagnosis of PDS.

PDS is a rare, but treatable cause of childhood intractable seizures. The most accepted view is that it is an autosomal recessive disease localized to chromosome $2 \mathrm{q} 31$ and the defect is in the enzyme glutamic acid decarboxylase (GAD) (1). However this theory is challenged by the recent reports of failure to detect mutation in GAD gene in a few families (2). Definite cases are defined as neonates, infants, or young children with recurrent seizures of any type that cease within seven days of the administration of oral pyridoxine, that recur when pyridoxine supplementation is withdrawn, and that cease again when pyridoxine is given (2). Our case fulfilled the criteria for definite PDS. The usual age of onset is in infancy. Untreated cases could result in permanent mental handicap (3). Though seizures started at the age of 2 years, our child had developmental delay since infancy. An initial partial response to antiepileptic drugs is well documented in PDS (2). There was mild reduction in seizure frequency with antiepileptic drugs in this child. We used a lower dose than recommended by Baxter et al. (3) in the diagnostic criteria. There could be individual variation in the dose of the vitamin required to control the seizures $(3,4)$. Good control of seizures has been reported with small dose of the vitamin also $(0.05-0.16 \mathrm{mg} / \mathrm{kg} /$ day $)(3,5)$. It is hypothesized that the individual variation in the dose of pyridoxine required to control the seizures 
is related to the individual variation in the level of enzyme activity (4).

This report highlights the clinical importance of a therapeutic trial of pyridoxine in all intractable seizures of early childhood. Though the age limit recommended was 18 months initially, with increasing number of cases identified in older children, it is recommended that all children below 3 years with intractable seizures should receive a trial with pyridoxine $(1,2,4,6)$. The oldest reported case is a 6-year-old boy (6). This particular case and the current report demand that, the recommendation needs to be modified by increasing the age limit to 6 years. It may be that the degree of enzyme activity determines the age at which seizures would occur and that individuals with relatively high enzyme activity would present late in infancy or early childhood as cases of atypical PDS (8). Till the age of 2 years this boy had only developmental delay. Gupta et al. (8) speculated that some patients with PDS (with a low level of enzyme defect) might present only with developmental delay, without seizures (8). If this hypothesis is proven, such patients will constitute a potentially treatable subgroup among children with idiopathic mental retardation. Occurrence of seizures after infancy and the therapeutic response to relatively low dose of pyridoxine could suggest partial enzyme activity in our child.

\section{References}

1. Gospe SM. Pyridoxine-dependent seizures: findings from recent studies pose new questions. Pediatr Neurol 2002; 26: 181-185.

2. Baxter P. Epidemiology of pyridoxine-dependent and pyridoxine responsive seizures in the UK. Arch Dis Child 1999; 81: 431-433.

3. Baxter P, Griffiths P, Kelly T, Gardner-Medwin D. Pyridoxine-dependent seizures: demographic, clinical, MRI and psychometric features, and effect of dose on intelligence quotient. Dev Med Child Neurol 1996; 38: 998-1001.

4. Rajesh R, Girija AS. Pyridoxine-dependent seizures: a review. Indian Pediatr 2003; 40: 633-638.

5. Grillo E, da Silva RJM, Barbato F. Pyridoxinedependent seizures responding to extremely low dose pyridoxine. Dev Med Child Neurol 2001; 43: 413-415.

6. Bachman DS. Late onset pyridoxine dependent convulsion. Ann Neurol 1983; 14: 692-693.

7. Claus SP, Braun KPJ, Dorland L, Bourrez-Swart M, van Nieuwenhuizen $O$, de Koning TJ. Unexplained epileptic encephalopathy: consider and reconsider pyridoxine dependent seizures. J Pediatr Neurol 2003; 1: 51-53.

8. Gupta VK, Mishra D, Mathur I, Singh KK. Pyridoxine-dependent seizures: a case report and a critical review of the literature. J Paediatr Child Health 2001; 37: 592-596. 